\title{
Novel DiR and SPIO nanoparticles embedded PEG-PLGA nanobubbles as a multimodal imaging contrast agent
}

\author{
Binhua Luo ${ }^{\mathrm{a}, \mathrm{b}}$, Huajie Zhang ${ }^{\mathrm{c}}$, Xuhan Liu ${ }^{\mathrm{a}}$, Rong Rao ${ }^{\mathrm{a}}$, Yun $\mathrm{Wu}^{\mathrm{d}}$ and Wei Liu ${ }^{\mathrm{a}, *}$ \\ ${ }^{a}$ College of Life Science and Technology, Huazhong University of Science and Technology, Wuhan \\ 430074, China \\ ${ }^{b}$ College of Pharmacy, Hubei University of Science and Technology, Xianning, China \\ ${ }^{c}$ Wuhan Institute of Physical Education, Wuhan, 430079, China \\ ${ }^{d}$ Department of Biomedical Engineering, University at Buffalo, State University of New York, Buffalo, \\ NY 14260, USA
}

\begin{abstract}
Fluorescence dye DiR and superparamagnetic iron oxide nanoparticles (SPIONs) embedded in PEG-PLGA nanobubbles (DiR-SPIO-NBs) were produced using double emulsion method on a membrane of Shirasu porous glass (SPG). The nanobubbles encapsulated with DiR and SPIONs had a liquid core (perfluoropentane) and a PEG-PLGA shell. DiR-SPIO-NBs showed biocompatibility based on MTT cytotoxicity and hemolysis studies. The PFP encapsulated in the nanobubbles experienced phase transition under ultrasonic irradation. Nanobubbles dispersed well in saline over 3 months, and the relaxivity was $127.9 \mathrm{mM}^{-1} \mathrm{~s}^{-1}$, suggesting that it could be used as a contrast agent in MRI. The MR and fluorescence images in vivo demonstrated that the signal intensity in the spleen and liver was significantly enhanced with the treatment of nanobubbles. In addition, results of ultrasound images suggested that the nanobubbles had persistent contrast ability. In conclusion, nanobubbles could be utilized as an US/MRI/fluorescence contrast agent.
\end{abstract}

Keywords: Nanobubbles, ultrasound-triggered phase transition, multimodal contrast agent, ultrasound imaging, magnetic resonance imaging, fluorescence imaging

\section{Introduction}

Medical imaging technologies such as ultrasound (US) imaging, magnetic resonance imaging (MRI) and fluorescence imaging, were applied to diagnosing cancer. However, each of these technologies has certain advantages and disadvantages. Recently, multimodal imaging has attracted the interest of researchers, due to its ability to make up for the shortcomings of a single-imaging mode $[1,2]$. In previous studies, microbubbles encapsulated with fluorescent dyes or superparamagnetic iron oxide (SPIO) have been used as US/fluorescence or MRI/US contrast agents [3, 4]. The diameters of the microbubbles are usually several microns $(2 \sim 8 \mu \mathrm{m})$, causing them to be trapped in the blood pool and

\footnotetext{
* Address for correspondence: Wei Liu, College of Life Science and Technology, Huazhong University of Science and Technology, Wuhan 430074, China. Tel.: +86-27-87792147; Fax: +86-27-87792234; E-mail: wliu@hust.edu.cn.
} 
thus imposing severe limitations on ultrasonic imaging and drug/gene [5]. In recent years, nanoscale bubbles (nanobubbles) have been the subject of research regarding extravascular ultrasonic imaging and drug/gene delivery [6,7]. Nanobubbles effectively accumulate in the tumor interstitium due to the enhanced permeability and retention (EPR) effect, naturally enabling them to serve as promising contrast agents for tumor ultrasonic imaging and treatment [8].

In previous studies, the preparation methods of nanobubbles are similar to those of microbubbles. These similar techniques include the mechanical stirring method, the ultrasonic treatment method, the freeze-drying method, the film hydration method, and the double emulsion-solvent evaporation method. These procedures are not well-controlled by the particles size. Furthermore, these methods use large amounts of organic solvents or strong mechanical stirring force, resulting in the encapsulated biomolecules losing their bioactivities [9].

In this study, uniform-sized poly (ethylene glycol)-poly (lactide-co-glycolide) (PEG-PLGA) nanobubbles encapsulated with DiR (1,1'-dioctadecyltetramethyl indotricarbocyanine ioide) and SPIO nanoparticles (DiR-SPIO-NBs) were produced using double emulsion method. The physi-chemical characteristics, the ultrasound-triggered phase transition, the biocompatibility and the utilization of nanobubbles as an US/MRI /fluorescence contrast agent were studied.

\section{Materials and methods}

\subsection{Materials}

PEG-PLGA (LA:GA=50:50 and molecular weight of 35,000 Da) was obtained from Shangdong Institute of Medical Instrument (Shangdong, China). PVA-217 (hydrolysis degree of $88.5 \%$ ) was purchased from Kuraray (Tokyo, Japan). 1, 1'-dioctadecyltetramethyl indotricarbocyanine ioide (DiR) was obtained from Biotium, Inc (Hayward, CA, USA). Superparamagnetic iron oxide (SPIO) nanoparticles $(10 \mathrm{~nm})$ were synthesized by our laboratory as reported by $\mathrm{Xu}$, et al. [10]. Perfluoropentane was purchased from Sigma (Sigma-Aldrich, USA). SPG membrane (the diameter of $0.6 \mu \mathrm{m})$ was purchased from Kiyomoto Iron Works Co. (Sadowara, Japan).

\subsection{Preparation of DiR-SPIO-NBS}

The premix membrane emulsification and solvent evaporation method was used to prepare DiR-SPIO-NBs. DiR $(1 \mathrm{mg})$, PEG-PLGA $(0.1 \mathrm{~g})$ and SPIO $(50 \mathrm{mg})$ were first dissolved in the THF solution $(5 \mathrm{~mL})$. PFP $(1 \mathrm{~mL})$ was added to the solution, followed by a high shearing at $10000 \mathrm{rpm}$ for about 4 min using homogenizer (Shanghai, China) under the low temperature in darkness to form $\mathrm{O}_{1} / \mathrm{O}_{2}$ primary emulsion. $35 \mathrm{~mL}$ saline and $0.3 \%$ PVA solution $(\mathrm{w} / \mathrm{v})$ were poured into the primary emulsion and stirred mechanically for about 2 min to form $\mathrm{O}_{1} / \mathrm{O}_{2} / \mathrm{W}$ double emulsion. The $\mathrm{O}_{1} / \mathrm{O}_{2} / \mathrm{W}$ double emulsion passed through SPG membrane equipment five times with 106 psi of nitrogen pressure at room temperature. Organic solvents were removed by evaporation, and the nanobubbles were formed after solidification. The nanobubble dispersion was centrifuged (10000 rpm, $10 \mathrm{~min})$ to obtain nanobubble precipitates, which were then dispersed in a normal saline solution. The samples were then stored at $4^{\circ} \mathrm{C}$ for further study.

\subsection{Characterization of DiR-SPIO-NBS}


The hydrodynamic size and the polydispersity index (PDI) of DiR-SPIO-NBs were measured on a Nano-ZS90 zetasizer (Malvern, UK) at $25^{\circ} \mathrm{C}$ using He-Ne laser of $633 \mathrm{~nm}$. To determine the zeta potential, the nanobubbles were diluted with ultrapure water until the conductivity of the dilute suspension was $40-50 \mu \mathrm{S} / \mathrm{cm}$. The morphology of DiR-SPIO-NBs was examined by TEM (Tecnai G2 20, FEI, USA).

In the stability experiment, DiR-SPIO-NBs were stored at $4{ }^{\circ} \mathrm{C}$ for 3 months. Changes in particle size and zeta potential were examined during the storage period $(0,0.5,1$, and 3 months).

\subsection{Imaging study in vitro}

\subsubsection{MR imaging}

To assess the $\mathrm{T}_{2}$ relaxation times, the nanobubbles were diluted with saline until the concentrations were $0.32 \%, 0.48 \%, 0.64 \%$ and $0.80 \%(\mathrm{~m} / \mathrm{m})$. Samples were examined on a $3.0-\mathrm{T}$ whole-body MR scanner (MAGNETOM Trio, A Tim System 3T, Siemens, Munich, Germany) with an 8-channel wrist joint coil. The parameters were optimized as follows: field of view $=120 \mathrm{~mm}$, base resolution $=384 \times 384$, slice thickness $=1.5 \mathrm{~mm}$, multiple echo times $=20,40,60,80,100,120,140 \mathrm{~ms}$, repetition time 2000 $\mathrm{ms}$, and scan time about 13-14 min. $\mathrm{T}_{2}$ relaxation rates were plotted against iron concentrations in the dilutions. Relaxivity was determined through a linear fitting model.

\subsubsection{US imaging}

The nanobubbles solution was added to the latex finger cot, positioned in a degassed water bath. The ultrasound contrast results were obtained from a B-mode clinical ultrasound imaging system (Philips Medical Systems, USA). To collect ultrasonic images, the $10 \mathrm{MHz}$ probe of a clinical ultrasound imaging system (L12-1) was immersed in the liquid. All contrast images were recorded in the B-mode, with frame rate of $37 \mathrm{~Hz}$ and Mechanical Index (MI) of 0.07.

\subsection{In vivo imaging study}

\subsubsection{In vivo $M R I$}

Rabbits afflicted with in situ VX2 liver cancer were anaesthetized with $10 \%$ of chloral hydrate $(0.4$ $\mathrm{mL} / 100 \mathrm{~g}$ ), then placed in the magnetic resonance imaging system at the prone position, and scanned before and after injection of nanobubbles $(1 \mathrm{~mL})$ through the ear vein. The scanning parameters were as follows: Field of view $=200 \mathrm{~mm}$, base resolution $=320 \times 320$, slice thickness $=4 \mathrm{~mm}$, echo time $=87$ $\mathrm{ms}$, repetition time $=1000 \mathrm{~ms}$, and scanning time $=39 \mathrm{~s}$.

\subsubsection{In vivo US imaging}

The anaesthetized rabbits were injected with $1 \mathrm{~mL}$ of the nanobubbles through the ear vein. The probe $(10 \mathrm{MHz})$ was used to obtain the tumor imaging. Sonovue microbubbles (Bracco, Italy), a commercial ultrasonic contrast agent, are used as control. The rabbits were sacrificed after completion of the experiments, and perfused with saline followed by $4 \%$ paraformaldehyde. The liver was removed and the tumor was separated, both of these were studied using an IVIS Lumina XR system (Caliper Life Sciences, USA). The ex vivo fluorescent images of the organs were acquired and analyzed. Finally, the organs were treated with hematoxylineeosin (H \& E) staining.

\subsubsection{In vivo fluorescence imaging}

The anesthetized BALB/c nude mice with HepG2 tumor were injected with a dose of $0.2 \mathrm{~mL}$ 
DiR-SPIO-NBs. The mice were then placed into the IVIS Lumina XR system in the ventral positions. The fluorescence images were acquired at $10 \mathrm{~min}, 30 \mathrm{~min}, 1 \mathrm{~h}, 2 \mathrm{~h}, 4 \mathrm{~h}, 6 \mathrm{~h}, 8 \mathrm{~h}, 11 \mathrm{~h}$ and $31 \mathrm{~h}$ after injection, respectively. The parameters as follows: exposure time $=$ auto, $\mathrm{f} / \mathrm{stop}=2$, binning $=4$, and $\mathrm{FOV}=10 \mathrm{~cm}$. After fluorescence imaging experiments, the major organs were separated, which would be analyzed by the IVIS Lumina XR system.

\section{Results and discussion}

\subsection{Characterization of DiR-SPIO-NBS}

The size/size distribution and TEM image of DiR-SPIO-NBs were shown in Figure 1. DiR-SPIO-NBs has a uniform size of $302.7 \pm 6.4 \mathrm{~nm}$ with PDI of $0.124 \pm 0.032$, and zeta potential of $-8.75 \pm 0.26 \mathrm{mV}$. TEM image indicated that the DiR-SPIO-NBs were core-shell spherical nanoparticles. The lipophilic SPIO and DiR were encapsulated in the polymer shell, while the PFP was in the core of the nanobubbles. The particle size and zeta potential measurements revealed that DiR-SPIO-NBs remained stable for over 3 months when stored in an aqueous solution at $4{ }^{\circ} \mathrm{C}$. DiR-SPIO-NBs showed good biocompatibility based on MTT cytotoxicity of HepG2 or HL-7702 cells and hemolysis studies.

A novel method using premix membrane emulsification and solvent evaporation was developed for the preparation of nanobubbles and other nanocarriers [11]. Unlike the conventional methods, which usually result in instability and uneven nanocarrier size [12], the nanobubbles prepared in this study are stable and uniform.

\subsection{In vitro imaging study}

MRI in vitro of nanobubbles was studied using a 3T MRI instrument. MR images of various iron concentrations were obtained with a $\mathrm{T}_{2}$-weighted sequence. The DiR-SPIO-NBs could reduce $\mathrm{T}_{2}$ significantly. Also, the $T_{2}$ relaxation corresponded with the iron concentration. These suggested that the nanobubbles could be used as a $\mathrm{T}_{2}$ contrast agent.

At room temperature, the PFP maintained its liquid state because its boiling point is $29^{\circ} \mathrm{C}$. The transition from liquid to gas occurred when the nanobubbles were activated by the ultrasound at $25^{\circ} \mathrm{C}$. The results showed that the DiR-SPIO-NBs exhibited phase transition behavior in response to the ultrasonic wave. Many bright spots appeared in the latex finger cot, which reflected the enhancement

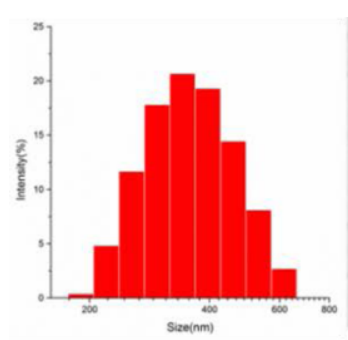

A

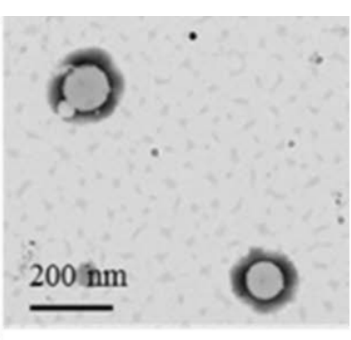

B

Fig. 1. The size distribution and morphology of DiR-SPIO-NBs. (A) Size distribution; (B) TEM image.

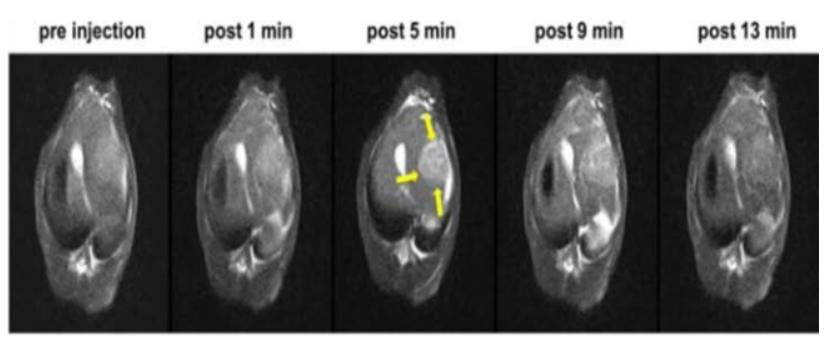

Fig. 2. T2-weighted MR images of rabbit liver bearing VX2 tumor with injection of DiR-SPIO-NBs. 
of echogenic movement signals. Naturally, this observation confirmed that the nanobubbles possessed acoustical activity [8].

\subsection{In vivo imaging study}

\subsubsection{In vivo $M R I$}

To evaluate whether or not the nanobubbles could act as an MRI contrast agent, rabbit liver were examined using a 3T MRI instrument with $\mathrm{T}_{2}$ sequence. As shown in Figure 2, before the nanobubble injection, the liver tumor boundaries were indistinct, while after injection, the boundaries gradually clear as time goes on. The signal strength reached its maximum at $5 \mathrm{~min}$, after, it began to decrease.

\subsubsection{In vivo ultrasound imaging}

The liver tumors could be recognized as low echoic mass. As shown in Figure 3, before the injection of the DiR-SPIO-NBs, the delineation and the boundaries were unclear, since the lesion was almost isoechoic with the normal tissue. After the injection, the entire liver and tumor rim were enhanced, the entirety of the tumors was enhanced within $5 \mathrm{~min}$. The signals did not decrease significantly in the tumor within $17 \mathrm{~min}$. The H\&E staining result confirmed that the tumor cells were of a large volume, and an irregular shape, exhibiting a hypertrophy of the nucleus and visible nuclear division.

\subsubsection{In vivo fluorescence imaging}

The fluorescence images in tumor-bearing nude mice were shown in Figure 4. After the injection of the DiR-SPIO-NBs, the tissue distribution of the fluorescence in vivo was obtained. At $10 \mathrm{~min}$ after injection, the signals were strongest, and continued to be for more than $11 \mathrm{~h}$. The fluorescence intensity ex vivo at $11 \mathrm{~h}$ after injection showed that higher quantities of DiR accumulated in the liver, spleen, and tumor more so than in other organs.
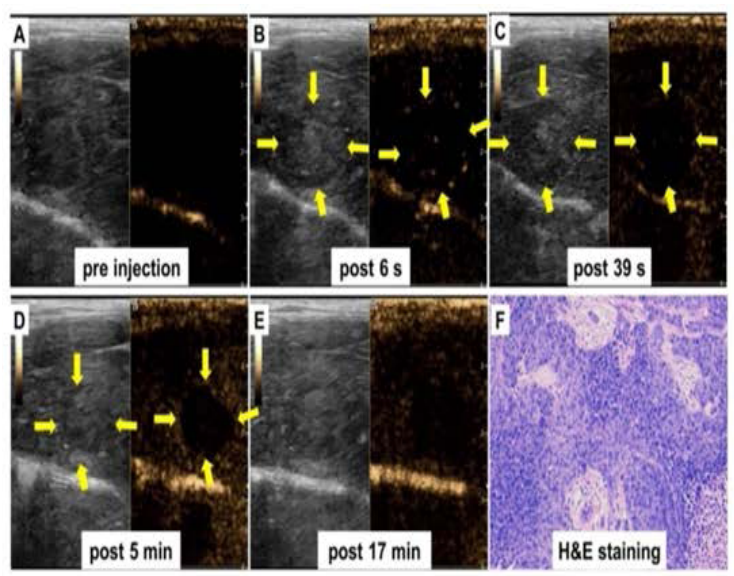

Fig. 3. Ultrasound images of rabbits liver bearing VX2 tumor before and after injection of DiR-SPIO-NBs. (A) - (E) Ultrasound images. (F) H\&E staining of tumor cell $(200 \times)$.

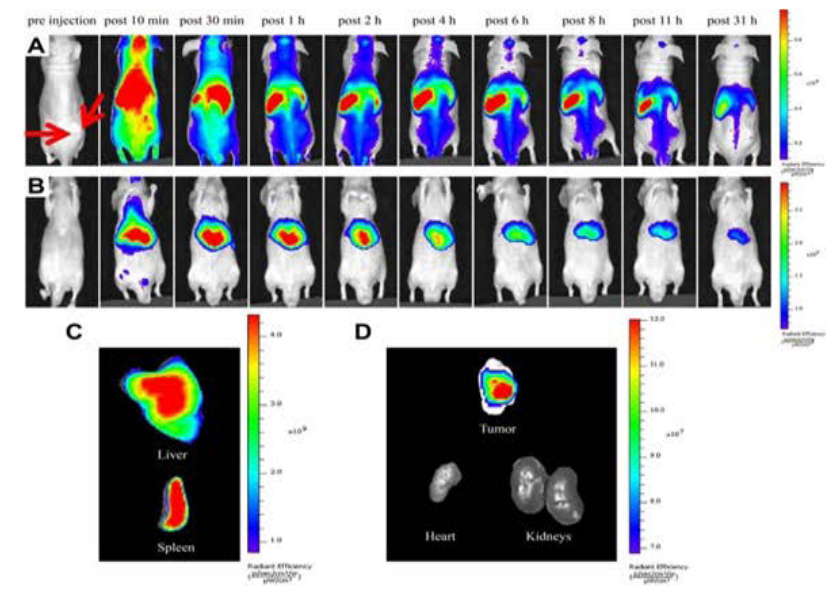

Fig. 4. Fluorescence imaging of nude mice bearing HepG2 tumor with injection of DiR-SPIO-NBs. (A) Dorsal views; (B) Ventral views; (C) Ex vivo images of liver and spleen; (D) Ex vivo images of tumor, heart and kidneys. 


\section{Conclusion}

Uniform-sized and stable DiR and SPIO nanoparticles embedded in PEG-PLGA nanobubbles were successfully prepared with the premix membrane emulsification and solvent evaporation method. The results from the in vitro and in vivo MRI, ultrasound images, and fluorescence images revealed that the nanobubbles had strong, long-lasting tumor-targeting and imaging contrast enhanced abilities. In conclusion, the results suggest that nanobubbles could be utilized as an US/MRI / fluorescence contrast agent for diagnosing cancer.

\section{Acknowledgment}

This work was financially supported by National Science Foundation of China (NSFC, 31170960 , 31470968) and National High Technology Research and Development Program of China (863 Program, 2012AA022704).

\section{References}

[1] D.E. Lee, H. Koo, I.C. Sun, et al. Multifunctional nanoparticles for multimodal imaging and theragnosis, Chemical Society Reviews 41 (2012), 2656-2672.

[2] H. Xing, W. Bu, S. Zhang, et al. Multifunctional nanoprobes for upconversion fluorescence, MR and CT trimodal imaging, Biomaterials 33 (2012), 1079-1089.

[3] R.X. Xu, J. Huang, J.S. Xu, et al. Fabrication of indocyanine green encapsulated biodegradable microbubbles for structural and functional imaging of cancer, Journal of Biomedical Optics 14 (2009), 034020.

[4] F. Yang, Y. Li, Z. Chen, et al. Superparamagnetic iron oxide nanoparticle-embedded encapsulated microbubbles as dual contrast agents of magnetic resonance and ultrasound imaging, Biomaterials 30 (2009), 3882-3890.

[5] S. Hernot and A.L. Klibanov, Microbubbles in ultrasound-triggered drug and gene delivery, Advanced Drug Delivery Reviews 60 (2008) 1153-1166.

[6] T. Yin, P. Wang, J. Li, et al. Ultrasound-sensitive siRNA-loaded nanobubbles formed by hetero-assembly of polymeric micelles and liposomes and their therapeutic effect in gliomas, Biomaterials 34 (2013), 4532-4543.

[7] H. Yang, W. Cai, L. Xu, et al. Nanobubble-affibody: Novel ultrasound contrast agents for targeted molecular ultrasound imaging of tumor, Biomaterials 37 (2014), 79-88.

[8] T. Yin, P. Wang, R. Zheng, et al. Nanobubbles for enhanced ultrasound imaging of tumors, International Journal of Nanomedicine 7 (2012), 895-904.

[9] W. Liu, X.L. Yang and W.S.W. Ho, Preparation of uniform-sized multiple emulsions and micro/nano particulates for drug delivery by membrane emulsification, Journal of Pharmaceutical Sciences 100 (2011), 75-93.

[10] Z.C. Xu, C.M. Shen, Y.L. Hou, et al. Oleylamine as both reducing agent and stabilizer in a facile synthesis of magnetite nanoparticles, Chemistry of Materials 21 (2009), 1778-1780.

[11] X. Cheng, H. Li, Y.C. Chen, et al. Ultrasound-triggered phase transition sensitive magnetic fluorescent nanodroplets as a multimodal imaging contrast agent in rat and mouse model, PLOS One 8 (2013), e85003.

[12] Z. Xing, J. Wang, H. Ke, et al. The fabrication of novel nanobubble ultrasound contrast agent for potential tumor imaging, Nanotechnology 21 (2010), 145607. 